\title{
A Low-Complexity Modulated Model Predictive Current Control Scheme for a PMSM based Starter Generator System for a More Electric Aircraft
}

\author{
Qi Wang, Student Member, IEEE, Haitao Yu, Member, IEEE, Chen Li, Student Member, IEEE, Seang \\ Shen Yeoh, Xiaoyu Lang Student Member, IEEE, Tao Yang, Senior Member, IEEE, Marco Rivera, \\ Senior Member, IEEE, Serhiy Bozhko, Senior Member, IEEE, and Patrick Wheeler, Senior Member, \\ IEEE
}

\begin{abstract}
Modulated model predictive control (M2PC) has recently emerged as a possible solution for control in starter generator systems in the more electric aircraft (MEA), due to its advantages of fixed switching frequency, fast response and good performance. However, conventional M2PC requires the prediction of each possible output voltage vector, which involves a heavy computational burden for the processor, especially for multilevel converters. This is an obstacle for practical industrial applications. To solve this problem this paper introduces a new, low-complexity modulated model predictive control (LC-M2PC) for a starter generator control system with a neutral point clamped (NPC) converter. The proposed LC-M2PC only needs prediction action once in each control interval, which can reduce the computational burden of processor. Fixed switching frequency is maintained and it can achieve a lower total harmonic distortion (THD) current than conventional M2PC, using space vector modulation (SVM). This proposed LC-M2PC method is validated on a prototype electrical starter generator (ESG) system test rig with three-level NPC converter. Experimental results verify the effectiveness of the proposed method.
\end{abstract}

Index Terms-Finite-control-set model predictive control (FCS-MPC), Model predictive control (MPC), modulated MPC (M2PC), permanent magnet machine (PMSM), more electric aircraft (MEA), low-complexity modulated model predictive control (LC-M2PC).

\section{INTRODUCTION}

$\mathrm{T}$ he aerospace industry is putting emphasis on the use of advanced technology to achieve more efficient and cleaner

This work has been submitted to the IEEE for possible publication. Copyright may be transferred without notice, after which this version may no longer be accessible.

Q. Wang, and H.T. Yu are with School of Electrical Engineering, Southeast University, Nanjing, 210096, China (e-mail: qwang.cn@outlook.com; htyu@ seu.edu.cn). (Corresponding author: Qi Wang).

C. Li, S.S. Yeoh, X.Y. Lang, T. Yang, S. Bozhko, and P. Wheeler are with the Power Electronics, Machines and Control Group, The University of Nottingham, Nottingham NG7 2RD, U.K (e-mail: chen.li@nottingham.ac.uk; seang.yeoh@nottingham.ac.uk;xiaoyu.lang@nottingham.ac.uk;tao.yang@not tingham.ac.uk;serhiy.bozhko@nottingham.ac.uk;pat.wheeler@nottingham.ac. uk).

M. Rivera is with the Department of Electrical Engineering, Universidad de Talca, Curico, 3341717,Chile (e-mail: marcoriv@utalca.cl). solutions. Due to the rapid development of power electronics, more electric aircraft (MEA) technology is a reality today [1]. MEA technology integrates the electric power generation, distribution and consumption of aircraft into a unified system [2]. One key technology for the MEA is the electrical starter generator (ESG) system [3]. Many machines can be considered for the ESG system, such as the switched reluctance machine (SRM), the induction machine (IM) and permanent magnet synchronous machine (PMSM) [4]. The SRM is highly robust and has a simple structure, but the output voltages are not sinusoidal and it also has a high torque ripple, which will influence the power quality and life time of the EGS system [5]. The IM has some mechanical constraints at high speed, such as stator expansion [6]. Compared with SRM and IM, the PMSM is more advantageous due to its high power density and light weight [1]. Therefore, this paper considers a PMSM and converter in an ESG control system. In a MEA, the complex operating scenarios of engine system requires the generators to have a wide speed range.

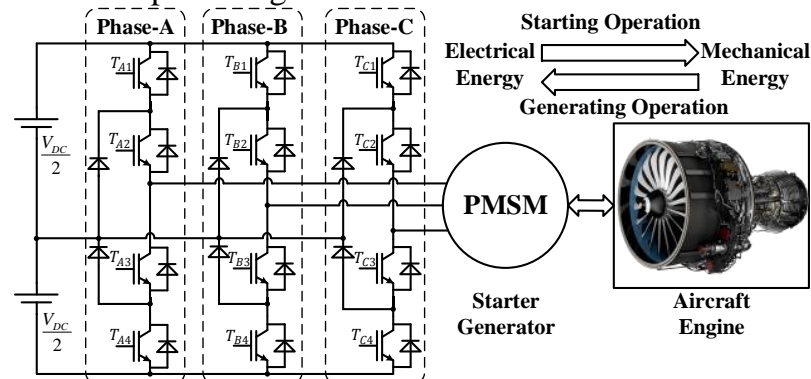

Fig.1 The structure diagram of ESG control system with NPC converter.

In order to guarantee a desirable carrier to noise ratio (CTNR), the high switching frequency is needed. However, for conventional high-power, two-level converters, it is hard to increase the switching frequency, due to thermal management. Silicon carbide (SiC) based two-level voltage source converters (VSC) have emerged in recent years [7]. SiC is a wide bandgap material, which offers potential benefits including higher efficiency, higher switching frequency, and higher temperature operation. However, a SiC based VSC is more expensive which limits its wide range of popularity and use [8]. To make sure that satisfactory power quality at low switching frequencies is 
maintained as well as consideration of economic benefits, using a conventional silicon $(\mathrm{Si})$ based multilevel converter is a good choice. This paper adopts a three-level neutral point clamped (NPC) converter in an ESG system, due to its lower switching losses and superior power quality compared to a two-level VSC [9]. The structure diagram of ESG is shown in Fig.1. The PMSM is connected to the aircraft engine by a mechanical shaft, and the DC bus voltage of NPC converter is $270 \mathrm{~V}$.

However, under different operating conditions, fast and stable control of the ESG is a challenging task. Proportional Integral (PI) based linear controllers for PMSMs are well-established. They offer simplicity, satisfactory dynamic and static performances with certain degree of tolerance of parameter. However, the ESG control system is a complex nonlinear system, and needs faster dynamic response, conventional PI control method is linear control method, difficult to resolve the contradiction between rapidity and stability [3]. The work points of PI controller need to be optimized in a real time. More and more nonlinear control methods have been proposed [10]-[13]. Due to a rapid increase in processor performance, the use of model predictive control (MPC) is possible as it offers multi-objective control and a fast dynamic response [14]. Finite-control-set model predictive control (FCS-MPC) has been proposed as a promising nonlinear control method for power electronic applications [14]-[16]. Due to its simple structure, easy implementation, and fast dynamic response, it has become very popular for power converters [17], [18] and motor drivers [19]-[21] in recent years. Compared to conventional linear controller (PI), FCS-MPC can include some constraints directly in cost function and has an ability of decouple [15]. However, as only one basic voltage vector is selected during one sample time in FSC-MPC, it has a variable switching frequency, producing a relatively large current ripple and total harmonic distortion (THD) of a low sampling frequency [22].

In order to get better control performance, a high sampling frequency is required in conventional FSC-MPC, which increases the computational burden of processor and causes increased switching losses. A high switching frequency is hard to implement in multilevel converter applications using low cost processors. A multi-level converter has a greater number of basic vectors and so the computational burden of processor is heavier. For example, a total of 27 basic voltage vectors (including eight redundant voltage vectors and eighteen non-redundant voltage vectors) are available in a three-level NPC converter [23]. All basic voltage vectors need to be predicted in each sampling period, a minimum time of 27 prediction is required, causing a heavy computational burden.

To solve the above problems, an improved FCS-MPC method with a modulator, named modulated model predictive (M2PC) was proposed by Tarisciotti et all [24]. M2PC has an intrinsic modulation scheme, which can produce a fixed switching frequency. M2PC has a higher switching frequency than FCS-MPC in same sampling period. M2PC current controller for a two-level voltage source inverter was introduced in [25]. Compared with conventional FSC-MPC, the power quality of the system has been improved. In [26], M2PC current controller for a brushless doubly fed induction machines was proposed, simulation and experimental results verifying the dynamic performance of the proposed method. M2PC for an active rectifier, a three-phase seven-level cascaded H-bridge back to back converter, a matrix converter have been proposed respectively in [24], [27], [28]. M2PC method for three-level NPC converter is first proposed by Rivera et all [29]. Subsequently, experimental results of an improved method using the redundant vectors to balance dc-link capacitor voltages was proposed [30]. However, a total of 27 vectors are available in the three-level NPC converter, which will also cause a high computational burden for the processor. Dwell times of synthesized vector are evaluated by a cost function in [29], which undermines the accuracy of synthesized vector.

To avoid these drawbacks, this paper presents a new low-complexity M2PC scheme with a three-level NPC converter for PMSM control. The propose LC-M2PC has advantages of predictive control method and also overcome disadvantages of conventional M2PC. The contributions of this paper are shown as follows:

1) The proposed LC-M2PC uses a predictive voltage vector to select sector in the phase plane, and reduces the number of prediction function calculations. The total calculation time is therefore shorter.

2) The principle of vector selection is introduced, analyzing the problem of the dwell times calculation method in conventional M2PC. The sector selection of modulator is decided by cost function and predictive voltages. Dwell times for each synthesized voltage vector is calculated according volt-second equilibrium equation, while the improvement of current quality for conventional $\mathrm{M} 2 \mathrm{PC}$ is verified.

3) For implementing proposed LC-M2PC effectively, the execution times of FCS-MPC, M2PC, and proposed method are calculated and compared.

The remainder of this paper will be structured as follows: Section II describes the conventional M2PC and its simplified form with a three-level NPC converter. Section III represents the proposed LC-M2PC scheme. In Section IV and Section V, simulation and experimental results are represented to verify the effectiveness of the proposed method. Finally, conclusions are drawn in Section VI.

\section{II.Conventional Modulated Model Predicative CURRENT CONTROL WITH NPC CONVERTER FOR MEA}

\section{A. Conventional M2PC with NPC for MEA}

The continuous mathematical model of a PMSM can be represented as:

$$
\left\{\begin{array}{l}
u_{d}=R_{s} i_{d}+L_{d} \frac{d i_{d}}{d t}-L_{q} \omega_{e} i_{q} \\
u_{q}=R_{s} i_{q}+L_{q} \frac{d i_{q}}{d t}+L_{d} \omega_{e} i_{d}+\psi_{m} \omega_{e}
\end{array}\right.
$$

Where, $u_{d}, u_{q}$ are the stator $d$ - $q$ coordinate axis voltages; $R_{s}$ is resistance of the stator. $L_{d}, L_{q}$ are the inductances of the stator in the stator $d-q$ coordinate axis; $\omega_{e}$ is electrical rotor speed and $\psi_{m}$ is the flux linkage of PMSM. From (1), a discrete time model can be derived by Euler discretization method. The sampling time is $T_{s}$, the discrete time model of the PMSM is: 


$$
\left\{\begin{array}{l}
u_{d}(k)=R_{s} i_{d}(k)+L_{d} \frac{i_{d}(k+1)-i_{d}(k)}{T_{s}}-L_{q} \omega_{e} i_{q}(k) \\
u_{q}(k)=R_{s} i_{q}(k)+L_{q} \frac{i_{q}(k+1)-i_{q}(k)}{T_{s}}+L_{d} \omega_{e} i_{d}(k)+\psi_{m} \omega_{e}
\end{array}\right.
$$

From (2), the next time prediction of the d-q axis currents can be found:

$$
\left\{\begin{array}{l}
i_{d}^{p}(k+1)=\left(1-\frac{R_{s} T_{s}}{L_{d}}\right) i_{d}(k)+\frac{L_{q} T_{s} \omega_{e}}{L_{d}} i_{q}(k)+\frac{T_{s}}{L_{d}} u_{d}(k) \\
i_{q}^{p}(k+1)=\left(1-\frac{R_{s} T_{s}}{L_{q}}\right) i_{q}(k)-\frac{L_{d} T_{s} \omega_{e}}{L_{q}} i_{d}(k)-\frac{\psi_{m} \omega_{e} T_{s}}{L_{q}}+\frac{T_{s}}{L_{q}} u_{q}(k)
\end{array}\right.
$$

Where, $i_{d}^{p}(k+1)$ and $i_{q}^{p}(k+1)$ are the $d-q$ axis predictive currents at $k+1$. In a conventional M2PC with an NPC converter for a PMSM control, three vectors are calculated by the cost function in each small sector. The cost function can be written as:

$$
g_{i}=\left(i_{d}^{*}-i_{d i}^{p}(k+1)\right)^{2}+\left(i_{q}^{*}-i_{q i}^{p}(k+1)\right)^{2} \quad i=0,1,2 .
$$

From (4), the dwell time for each vector can be computed as [29]:

$$
\begin{aligned}
& d_{0}=T_{s} g_{1} g_{2} /\left(g_{0} g_{1}+g_{1} g_{2}+g_{0} g_{2}\right) \\
& d_{1}=T_{s} g_{0} g_{2} /\left(g_{0} g_{1}+g_{1} g_{2}+g_{0} g_{2}\right) \\
& d_{2}=T_{s} g_{0} g_{1} /\left(g_{0} g_{1}+g_{1} g_{2}+g_{0} g_{2}\right) \\
& T_{s}=d_{0}+d_{1}+d_{2}
\end{aligned}
$$

With (5), the total cost function can be defined as :

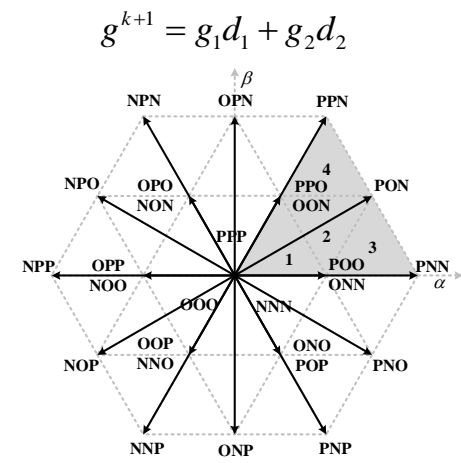

Fig. 2 The division sector diagram of M2PC for three-level NPC converter.

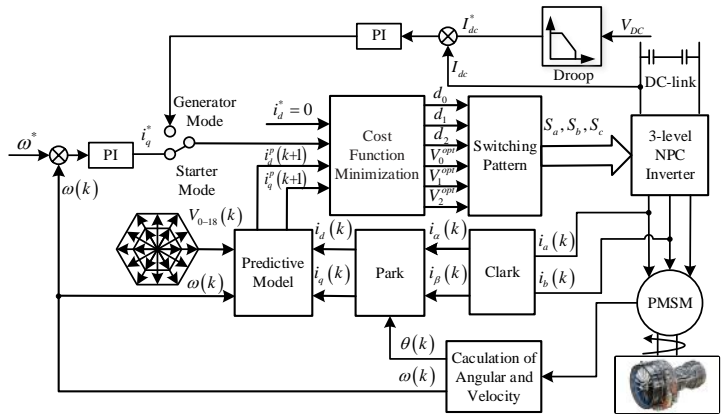

Fig.3 The control diagram of M2PC for ESG system of MEA with NPC converter.

It can be seen from the vector diagram of conventional M2PC, as shown in Fig.2, including six large triangular sectors. Each large triangular area is divided into four small triangular sectors. The optimal three vectors and dwell times, which minimize the total cost function, are selected by considering 24 small sectors. Further details of the M2PC method for a three-level NPC converter can be found in [29], [30]. Fig.3 shows the control structure of the M2PC.

\section{B. Simplified form of M2PC for an NPC Converter}

From Part A, for three-level NPC converter, it can be seen that conventional M2PC needs to consider 24 small sectors in each sample period. In each small sector, three voltage vectors are taken in to equation (3) to obtain predictive currents. Therefore, the total calculation of equation (3) is 72 (three vectors in one small sector, not including redundant vectors). The total calculation of cost function as shown in equation (4) is still 72 in each sample period, causing a high computational burden [29].

An effective method for FCS-MPC algorithm simplification was proposed in [31], using predictive voltages instead of predictive currents. The performance of this "single predictive" method is same as that of the conventional FCS-MPC. Therefore, this method of simplification also can be used to improve conventional M2PC algorithm. Predicting the $d-q$ axis voltages:

$$
\left\{\begin{array}{l}
u_{d}^{p}(k)=R_{s} i_{d}(k)+L_{d} \frac{i_{d}^{*}(k+1)-i_{d}(k)}{T_{s}}-L_{q} \omega_{e} i_{q}(k) \\
u_{q}^{p}(k)=R_{s} i_{q}(k)+L_{q} \frac{i_{q}^{*}(k+1)-i_{q}(k)}{T_{s}}+L_{d} \omega_{e} i_{d}(k)+\psi_{m} \omega_{e}
\end{array}\right.
$$

Where, and are the d-q axis voltage predictions. As shown in equation (7), the number of executions of (7) is only 1 in this simplified M2PC in each sample period, which called "single predictive", reducing computational burden of processor effectivly. Different from conventional M2PC, the cost function of this simplified M2PC is a calculation of the distance between predictive voltage and the voltage of the basic vector in each small sector. The cost function can be written as:

$$
g_{i}=\left(u_{d}^{p}(k)-u_{d i}(k)\right)^{2}+\left(u_{q}^{p}(k)-u_{q i}(k)\right)^{2} \quad i=0,1,2 .
$$

Where, and are d-q axis voltages, which are calculated by basic vectors. Only three basic vectors in one sector, so $i=0,1,2$. From (8), the dwell time for each vector can be computed from equation (5) and equation (6) [29]. This simplified form of the M2PC (S-M2PC) can save most of the calculation time of the processor.

\section{PRINCIPLE OF PROPOSED LC-M2PC METHOD}

From above contents, it can be seen that the number of executions of (7) is only 1 in S-M2PC in each sample period, reducing computational burden of processor effectivly. However, the total calculation of cost function as shown in equation (8) is still 72. To further save execution time of processor as well as improve the current quility, this part propose a new low-complexity M2PC scheme with a three-level NPC converter for PMSM control system.

\section{A. Large Sector Selection}

Different from conventional M2PC, sector division in the proposed LC-M2PC adopts a hexagon division method, conversing three-level to two-level in each small sector. The 
method of sector division refers to Fig.4, including six large hexagons which includes seven mapped vectors and each hexagon has six small triangular sectors.

Same as S-M2PC method, the predicted d-q axis voltages are calculated using (7). The number of executions of (7) is still 1 in each sample period. Then, the predicted stationary reference voltages can be formed using an inverse Park transformation:

$$
\left[\begin{array}{l}
u_{\alpha}^{p}(k) \\
u_{\beta}^{p}(k)
\end{array}\right]=\left[\begin{array}{cc}
\cos \theta & -\sin \theta \\
\sin \theta & \cos \theta
\end{array}\right]\left[\begin{array}{l}
u_{d}^{p}(k) \\
u_{q}^{p}(k)
\end{array}\right]
$$

According to predicted stationary reference voltages in equation (9), the large hexagon sector is decided by (10) and Table I.

$$
\begin{cases}u_{\alpha}^{p}>0 & A=1, \text { otherwise }, A=0 \\ \frac{\sqrt{3}}{3} u_{\alpha}^{p}+u_{\beta}^{p}>0 & B=1, \text { otherwise, } B=0 \\ u_{\beta}^{p}-\frac{\sqrt{3}}{3} u_{\alpha}^{p}>0 & C=1, \text { otherwise, } C=0 \\ N=4 A+2 B+C & \end{cases}
$$

In selected large hexagon sector, it has six small triangular sectors which is same as conventional diagram of two-level voltage vector. The method of selecting small triangular sectors is described in detail in Part B.

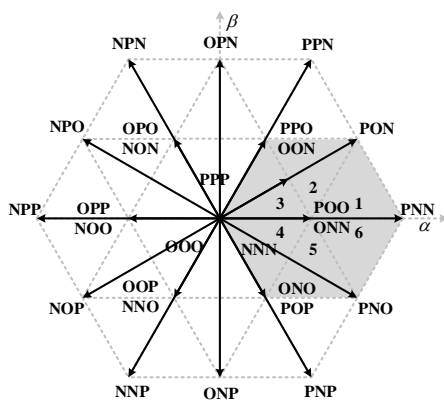

Fig. 4 The division large sector diagram of proposed LC-M2PC for three-level NPC converter.

TABLE I

SECTOR DIVISION OF PROPOSED METHOD

\begin{tabular}{ccccccc}
\hline \hline$N$ & 0 & 1 & 3 & 4 & 6 & 7 \\
\hline Sector number & 5 & 4 & 3 & 6 & 1 & 2 \\
\hline
\end{tabular}

\section{B. Small Sector Vector Selection}

After the selection of a large sector, the three-level can be simplified to two level in the selected large hexagon sector. Predictive stationary reference voltages in selected large hexagon sector can be derived from (11).

$$
\left\{\begin{array}{l}
u_{2 \alpha}^{p}(k)=u_{\alpha}^{p}(k)-\frac{1}{3} \cos \left((s-1) * \frac{\pi}{3}\right) \\
u_{2 \beta}^{p}(k)=u_{\beta}^{p}(k)-\frac{1}{3} \sin \left((s-1) * \frac{\pi}{3}\right)
\end{array}\right.
$$

Where, $\quad u_{2 \alpha}^{p}(k)$ and $u_{2 \beta}^{p}(k)$ are predicted stationary reference voltages in the selected large hexagon sector, $\mathrm{s}$ is the number of large hexagon sector, $s=1,2 \ldots 6$. Therefore, the predicted d-q axis voltages in the selected large hexagon sector can be calculated using Park transforms:

$$
\left[\begin{array}{l}
u_{2 d}^{p}(k) \\
u_{2 q}^{p}(k)
\end{array}\right]=\left[\begin{array}{cc}
\cos \theta^{\prime} & \sin \theta^{\prime} \\
-\sin \theta^{\prime} & \cos \theta^{\prime}
\end{array}\right]\left[\begin{array}{c}
u_{2 \alpha}^{p}(k) \\
u_{2 \beta}^{p}(k)
\end{array}\right]
$$

Where, $u_{2 d}^{p}(k)$ and $u_{2 q}^{p}(k)$ are the d-q axis voltages in the selected large hexagon sector. $\theta^{\prime}$ can be calculated as $u_{2 \alpha}^{p}(k)$ and $u_{2 \beta}^{p}(k)$. In Fig.5, using first large hexagon sector as an example, the distance of two adjacent vectors and predictive voltage vector is calculated using (13).

$$
g_{k}=\left(u_{2 d}^{p}(k)-u_{d 2 k}(k)\right)^{2}+\left(u_{2 q}^{p}(k)-u_{q 2 k}(k)\right)^{2} k=1,2 .
$$

$u_{d 2 k}(k)$ and $u_{q 2 k}(k)$ are d-q axis voltages of two adjacent vectors. The total cost function can be defined as:

$$
g_{\text {total }}=g_{1}+g_{2}
$$

After considering six small sectors shown in Fig. 5, two adjacent voltage vectors, minimizing the total cost function, are selected. For example, in first small sector, predictive voltage vector will be composed of PON, PNN and POO. If the prediction voltage vector in center of hexagon sector, the dwell times of these two adjacent voltage vectors are 0 . Therefore, there is a possibility that the prediction voltage vector in the center of large hexagon sector. The dwell times for proposed LC-M2C are calculated by these two adjacent voltage vectors, which will be introduced in detail at next section.

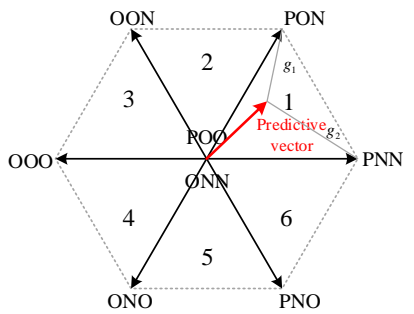

Fig. 5 The division small sector diagram in first large hexagon sector.

\section{Calculation of the Dwell Times}

For example, as shown in Fig.7, the predictive voltage vector is $u_{s}$, the end point of predictive voltage vector is right in the center of equilateral triangle that consists of basic $v_{1}, v_{2}$ and $d_{v_{1}-v_{2}}$ (difference between $v_{1}$ and $v_{2}$ ). The angle between $u_{s}$ and the basic vector $v_{1}$ is $30^{\circ}$.

From equation (8), it can be seen that the cost function is $g_{k}=\left(u_{2 d}^{p}(k)-u_{d 2 k}(k)\right)^{2}+\left(u_{2 q}^{p}(k)-u_{q 2 k}(k)\right)^{2}$, which is the distance between basic vector and predictive voltage vector. Where, $k=0,1,2$ corresponds to $v_{0}, v_{1}, v_{2}$. Therefore, the distances of predictive voltage vector $u_{s}$ to $v_{1}, v_{2}, v_{0}$ are same as $g_{1}, g_{2}, g_{0}$. The end of predictive voltage vector $u_{s}$ is at the center of an equilateral triangle, so $g_{0}=g_{1}=g_{2}$. The dwell times of this simplified M2PC are calculated according to (5), then equation (15) is derived:

$$
d_{0}=d_{1}=d_{2}=\frac{1}{3} T_{s}
$$

The dwell times of $v_{1}, v_{2}, v_{0}$ are same. This is because that the calculation of dwell times is directly proportional in the cost functions for a conventional M2PC as well as S-M2PC. The calculation of dwell times directly by linear proportional relation is not accurate, following is comparative explanations with convention volt-second equilibrium method. 
The same assumption that the end point of predictive voltage vector $u_{s}$ is right in the center of equilateral triangle. Dwell times are calculated by volt-second equilibrium method, which are same as conventional two-level space vector modulation (SVM), calculated by (16) in every small sector.

$$
\begin{aligned}
& d_{1}=\frac{\sqrt{3}\left|u_{s}\right|}{\left|V_{D C}^{\prime}\right|} T_{s} \sin \left(\frac{\pi}{3}-\theta\right) \\
& d_{2}=\frac{\sqrt{3}\left|u_{s}\right|}{\left|V_{D C}^{\prime}\right|} \sin (\theta) \\
& d_{0}=T_{s}-d_{1}-d_{2}
\end{aligned}
$$

Where, $V_{D C}{ }^{\prime}=V_{D C} / 2, V_{D C}$ is the DC bus voltage. $u_{s}$ is predictive voltage vector. For example, $\theta=30^{\circ}$, $\left|u_{s}\right|=\sqrt{3}\left|V_{D C}\right| / 12,\left|v_{1}\right|=\left|v_{2}\right|=\left|V_{D C}\right| / 3$ as shown in Fig.6. The dwell times for proposed LC-M2PC are calculated:

$$
\begin{aligned}
& d_{1}=\frac{\sqrt{3} \cdot \sqrt{3}\left|V_{D C}\right| / 12}{\left|V_{D C} / 2\right|} T_{s} \sin \left(\frac{\pi}{3}-\frac{\pi}{6}\right)=\frac{1}{2} T_{s} \\
& d_{2}=\frac{\sqrt{3} \cdot \sqrt{3}\left|V_{D C}\right| / 12}{\left|V_{D C} / 2\right|} T_{s} \sin \frac{\pi}{6}=\frac{1}{2} T_{s} \\
& d_{0}=T_{s}-d_{1}-d_{2}=0
\end{aligned}
$$

The dwell times of $\mathrm{v}_{1}, v_{2}, v_{0}$ that are calculated by the equations (17) are $T_{s} / 2, T_{s} / 2$, and 0 , respectively.

Therefore, the dwell times of a conventional M2PC are not accurate, which will cause error between the resultant vector and the predicted vector. In LC-M2PC, calculation of dwell times adopts a conventional volt-second equilibrium method.

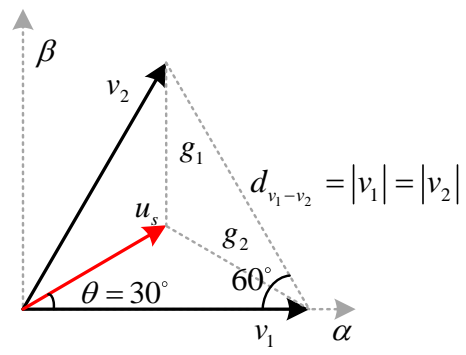

Fig. 7 The dwell times of predictive voltage vector.

\section{Simulation Results}

In order to validate the effectiveness of the proposal LC-M2PC method, four different methods are compared by simulation results. Method 1 is conventional FCS-MPC, Method 2 is the simplified form of M2PC (S-M2PC), and Method 3 denotes the proposed LC-M2PC. The simulation parameters of the PMSM are shown in Table II. The sample time of conventional FCS-MPC is $200 \mu s$. The sample time of other two methods are the same, set to $250 \mu \mathrm{s}$, and the interrupt frequency for other two methods is $4 \mathrm{kHz}$. The simulation results are for both steady state and transient conditions.

Fig. 8 shows the phase current responses of three methods at a reference speed $1000 \mathrm{rpm}$. The initial load is $0 N \cdot \mathrm{m}$, a load torque $2 \mathrm{~N} \cdot \mathrm{m}$ is applied at $0.15 \mathrm{~s}$. It is obvious that the phase current ripple for the conventional FCS-MPC scheme is higher than for the S-M2PC, and LC-M2PC schemes. The LC-M2PC has a lower phase current ripple than S-M2PC.

TABLE II

PARAMETER SETTING OF SIMULATION

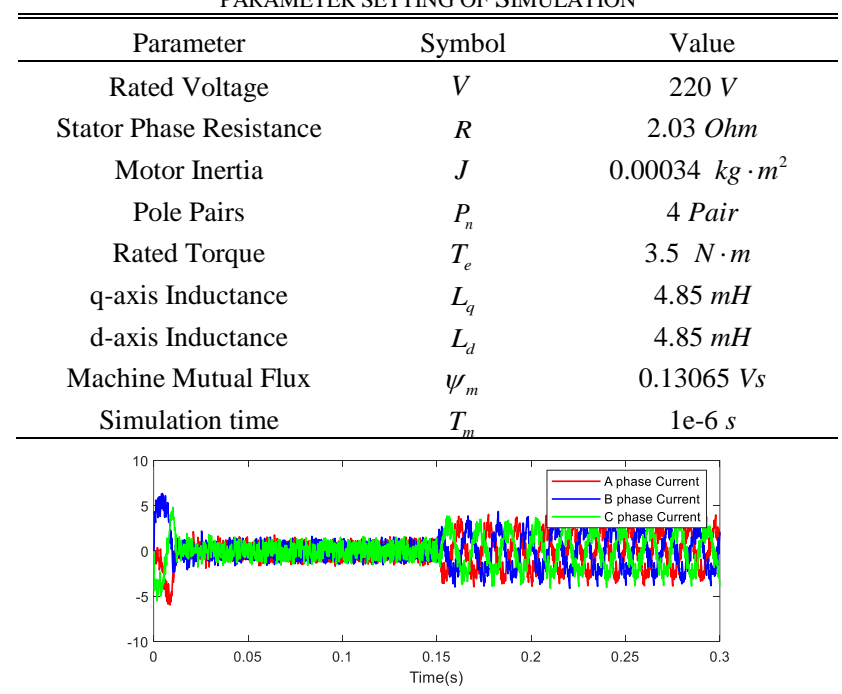

(a)

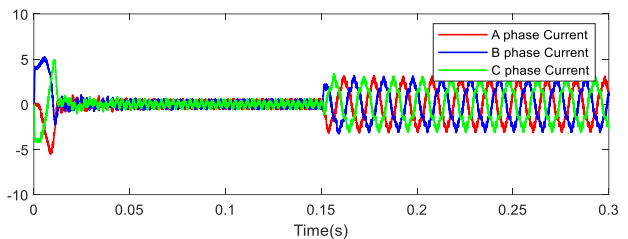

(b)

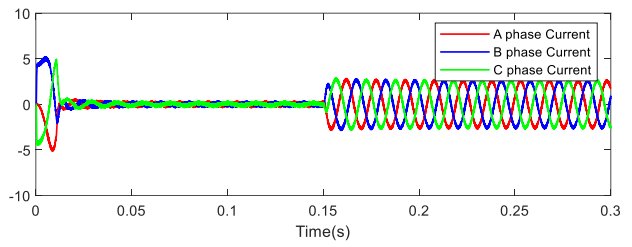

(c)

Fig. 8 Simulation results of phase currents. (a) FCS-MPC (b) S-M2PC (c) LC-M2PC.

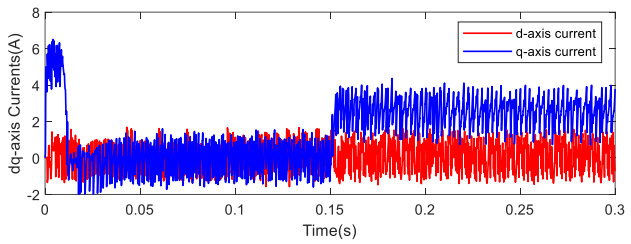

(a)

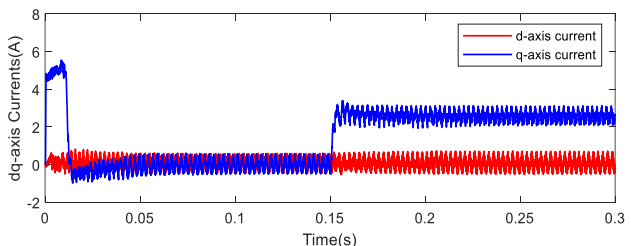

(b)

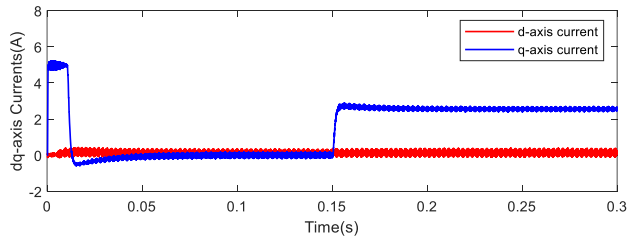

(c)

Fig. 9 Simulation results of d-q axis currents. (a) FCS-MPC (b) S-M2PC (c) LC-M2PC. 


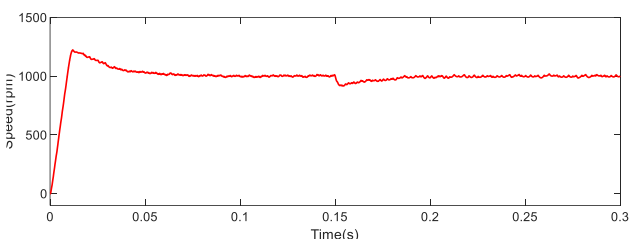

(a)

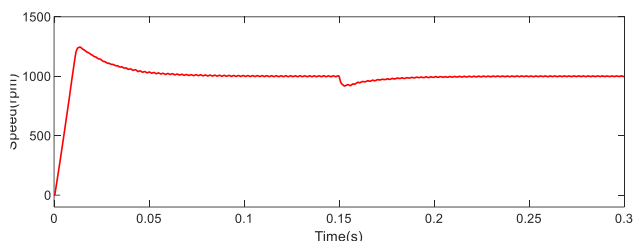

(b)

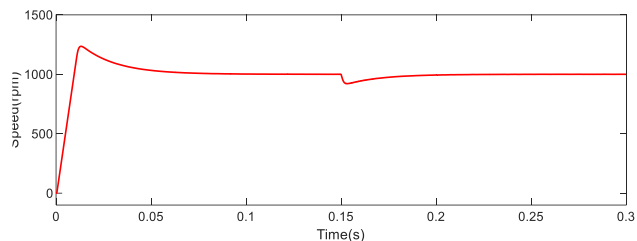

(c)

Fig. 10 Simulation results of speed. (a) FCS-MPC (b) S-M2PC (c) LC-M2PC.

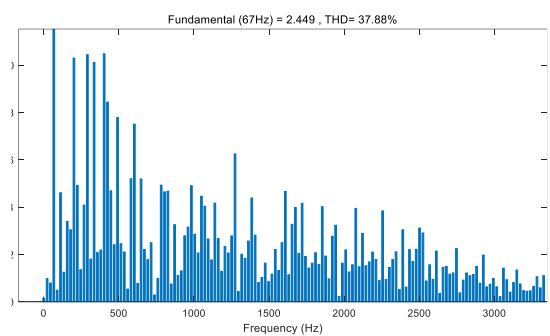

(a)

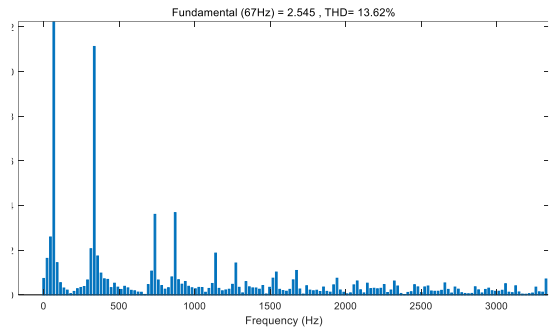

(b)

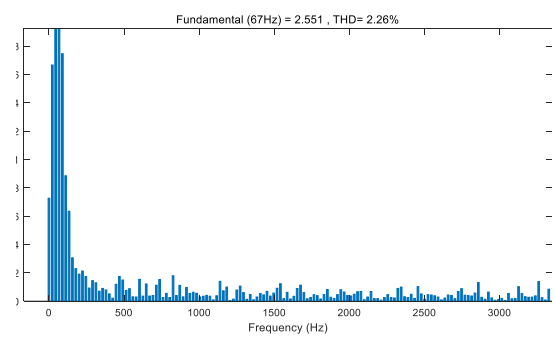

(c)

Fig. 11 The THD of A phase current at 1000rpm. (a) FCS-MPC (b) S-M2PC (c) LC-M2PC.

Figs. 9 and 10 show the simulation results of the $d$ - $q$ axis currents and speed. It can be seen that the speed is increasing at the beginning, and the $q$-axis current traces the reference $q$-axis current very well. Fig.11 shows the total harmonic distortion (THD) for these four different methods at 1000rpm. The THD for A phase current is analyzed over five cycles. The fundamental frequency is $67 \mathrm{~Hz}$, the maximum frequency is 50 times of the fundamental frequency. The THD for FCS-MPC, S-M2PC, LC-M2PC are $37.88 \%, 13.62 \%$, and $2.26 \%$ at $1000 \mathrm{rpm}$, respectively. It can be seen that the classic FCS-MPC has a higher THD than S-M2PC and LC-M2PC, because it has variable switching frequency. The classic FCS-MPC has lower average switching frequency than S-M2PC and LC-M2PC. The proposed LC-M2PC adopts conventional volt-second equilibrium method to calculate dwell times. Therefore, LC-M2PC has a better power quality than S-M2PC with the same fixed switching frequency. The simulation results are found to be mostly in agreement with the theories.

\section{EXPERIMENTAL RESULTS}

To verify the performance of the proposed LC-MPC, a prototype is implemented using a three-level NPC converter. The driver motor is a PMSM, and another PMSM is applied as load. The three-level NPC converter was built using IGBTs (Infineon, FF35R12RT4). The main control processor was a TMS320F28335 with a 150Mhz clock frequency. The maximum current limit was 5A. The control loop interrupt frequency of main program was $4 \mathrm{kHz}$, classic FCS-MPC, $\mathrm{S}-\mathrm{M} 2 \mathrm{PC}$ and LC-M2PC were programmed in C language.

To make S-M2PC available in $4 \mathrm{kHz}$ control frequency, redundant voltage vectors as well as neutral point balancing are not considered. The main purpose of this paper is to compare the control performance of these different methods. The execution time for each MPC method is shown in Table III. The cycle interrupt time of the control loop is $250 \mu \mathrm{s}$, which is the same as sampling time. It can be seen from Table III that only the execution time of conventional M2PC is $384 \mu s$, adding some other executable program, the total execution time is greater than $250 \mu \mathrm{s}$, which cannot complete the calculation in control cycle period. The S-M2PC needs less than half the execution time of conventional M2PC by "single prediction". The proposed LC-M2PC only needs $32 \mu s$, which therefore has ability to run at a higher control loop interrupt frequency. The parameters of PMSM are same as simulation, as shown in Table II.

TABLE III

EXECUTION TIME IN TMS320F28335

\begin{tabular}{rllll}
\hline \hline Method & FCS-MPC & M2PC & S-M2PC & LC-M2PC \\
\hline Execution time & $218 \mu \mathrm{s}$ & $384 \mu \mathrm{s}$ & $182 \mu \mathrm{s}$ & $32 \mu \mathrm{s}$ \\
\hline
\end{tabular}

A high sampling frequency is required for the FCS-MPC to maintain a good control [22]. However, due to the limits of the processor, conventional FCS-MPC cannot run at a high sampling frequency, especially for a multilevel converter. It can be clearly seen that the proposed LC-M2PC method has a much lower execution time and can run at a $15 \mathrm{kHz}$ sampling frequency. For comparison with other methods, the sampling frequency is uniformly set to $4 \mathrm{kHz}$.

Experiments have been completed to verify the dynamic performance and steady state response of the proposed LC-M2PC method. In order to verify the dynamic performance of the proposed LC-M2PC method, comparison experimental results of conventional S-M2PC, and LC-M2PC methods are also presented in Figs. 12-13.

The system runs at a stable speed, the target speed is changed 
suddenly. From Fig. 12, it is seen that the speed of PMSM decreases quickly to target speed. All methods have good dynamic performance, the system can get target speed quickly. However, S-M2PC has larger current and speed ripples than LC-M2PC. The reference speed is changing between 1600rpm and 400rpm, the actual speed of PMSM has ability to trace the reference speed within $100 \mathrm{~ms}$.

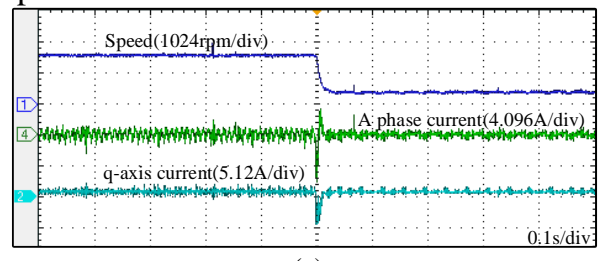

(a)

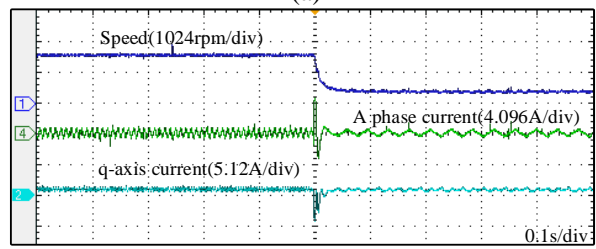

(b)

Fig. 12 Experimental responses in the case of speed decreasing. (a) S-M2PC (b) LC-M2PC

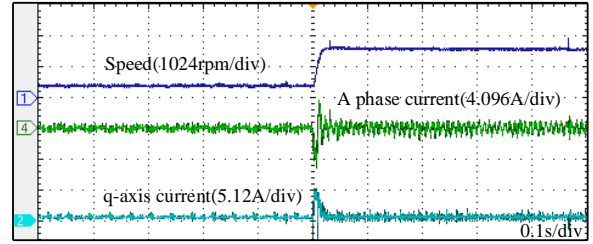

(a)

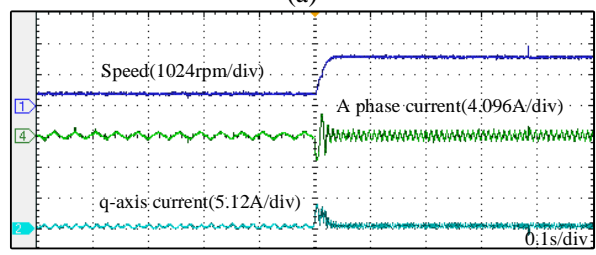

(b)

Fig. 13 Experimental responses for the case of a speed increasing. (a) S-M2PC (b) LC-M2PC.

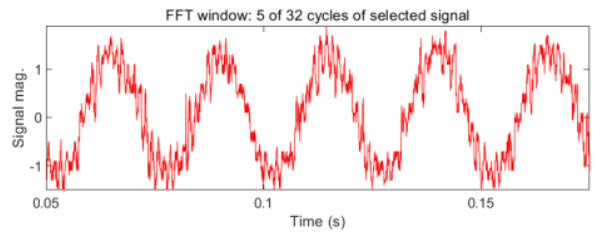

(a)

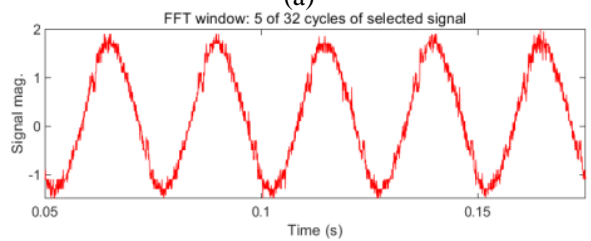

(b)

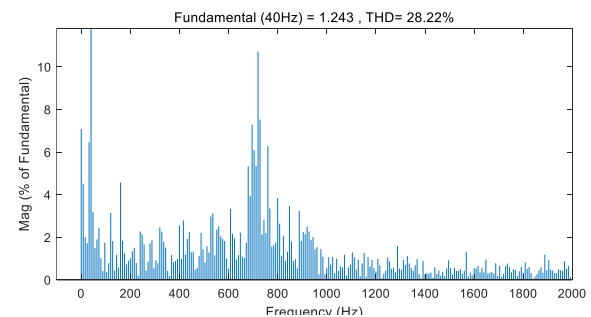

(c)

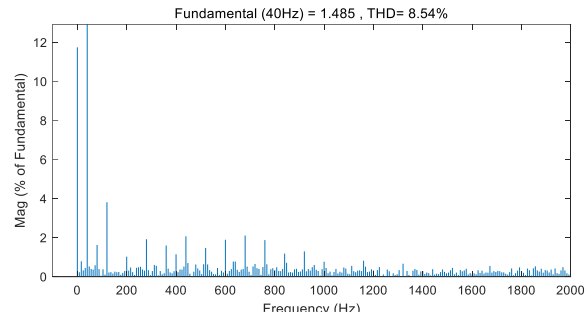

(d)

Fig. 14 Steady-state response and Harmonic spectrum at 600rpm. (a) S-M2PC (b) LC-M2PC (c) Harmonic spectrum of S-M2PC (d) Harmonic spectrum of LC-M2PC.

From Fig. 13, it is seen that the PMSM increases speed quickly to target speed. All methods have a good speed tracking performance. Fig.14 shows the steady-state response of both methods at 600rpm. The total harmonic distortion (THD) of A phase current of each method is calculated. The THD for S-M2PC, and LC-M2PC are $28.22 \%$ and $8.54 \%$, respectively. It can be seen that the proposed LC-M2PC and S-M2PC has same switching frequency. The proposed LC-M2PC method has a lower current ripple than S-M2PC method, because of the improving calculation of dwell times.

\section{CONCLUSIONS}

This paper presents a new LC-M2PC for a starter generator system with a three-level NPC converter for use in a MEA. The conventional M2PC method for three-level NPC converter is reviewed and the computation complexity and dwell times of M2PC analyzed. Then, a new LC-M2PC method with a fixed switching frequency was proposed. The proposed LC-M2PC method is implemented in an ESG test rig. The proposed method uses a predicted voltage vector to select the large hexagon sector directly, and only requires a single prediction action in each small sector. The execution time of proposed method is $32 \mu s$ in a TMS320F28335, which has the ability to run within a $20 \mathrm{kHz}$ control loop interrupt frequency. The dwell time calculation procedure of the proposed method adopts conventional SVM, which significantly improves the quality of the phase current. Finally, simulation and experimental results verify that the proposed method has satisfactory control performance and greatly reduces the computation burden of processor.

Compared to conventional M2PC, the proposed LC-M2PC method can obtain much better steady state ability while keeping similar dynamic performence. The structure of the proposed LC-M2PC is simple and easy to be implemented on low cost processors, which has a certain industrial application prospect.

\section{REFERENCES}

[1] F. Gao, X. Zheng, S. Bozhko, C. I. Hill, and G. Asher, "Modal Analysis of a PMSG-Based DC Electrical Power System in the More Electric Aircraft Using Eigenvalues Sensitivity," IEEE Transactions on Transportation Electrification, vol. 1, no. 1, pp. 65-76, Jun. 2015, doi: 10.1109/TTE.2015.2427312.

[2] X. Lang, T. Yang, C. Li, H. B. Enalou, S. Bozhko, and P. Wheeler, “A Dual-Channel-Enhanced Power Generation Architecture With Back-to-Back Converter for MEA Application," IEEE Transactions on 
Industry Applications, vol. 56, no. 3, pp. 3006-3019, May 2020, doi: 10.1109/TIA.2020.2974145.

[3] S. S. Yeoh, T. Yang, L. Tarisciotti, C. I. Hill, S. Bozhko, and P. Zanchetta, "Permanent-Magnet Machine-Based Starter-Generator System With Modulated Model Predictive Control," IEEE Transactions on Transportation Electrification, vol. 3, no. 4, pp. 878-890, Dec. 2017, doi: 10.1109/TTE.2017.2731626.

[4] M. E. Elbuluk and M. D. Kankam, "Potential starter/generator technologies for future aerospace applications," IEEE Aerospace and Electronic Systems Magazine, vol. 12, no. 5, pp. 24-31, May 1997, doi: $10.1109 / 62.587815$.

[5] S. S. Yeoh, "Control Strategies for the More Electric Aircraft Starter-Generator Electrical Power System," University of Nottingham, UK, 2016.

[6] R. Anbarasu et al., "Design and experimental investigation of high speed squirrel cage induction motor," in Proceedings of International Conference on Power Electronics, Drives and Energy Systems for Industrial Growth, Jan. 1996, vol. 2, pp. 920-926 vol.2, doi: 10.1109/PEDES.1996.536395.

[7] J. Colmenares, D.-P. Sadik, P. Hilber, and H.-P. Nee, "Reliability Analysis of a High-Efficiency $\mathrm{SiC}$ Three-Phase Inverter," IEEE Journal of Emerging and Selected Topics in Power Electronics, vol. 4, no. 3, pp. 996-1006, Sep. 2016, doi: 10.1109/JESTPE.2016.2551980.

[8] J. Rabkowski, D. Peftitsis, and H.-P. Nee, "Silicon Carbide Power Transistors: A New Era in Power Electronics Is Initiated," IEEE Industrial Electronics Magazine, vol. 6, no. 2, pp. 17-26, Jun. 2012, doi: 10.1109/MIE.2012.2193291.

[9] E. A. Kumar, R. S. Rao, and K. C. Sekhar, "Simplified model predictive control of a three-phase T-type NPC inverter," IET Power Electronics, vol. 12, no. 8, pp. 1917-1930, 2019, doi: 10.1049/iet-pel.2018.5673.

[10] G. Wang, X. Hao, N. Zhao, G. Zhang, and D. Xu, "Current Sensor Fault-Tolerant Control Strategy for Encoderless PMSM Drives Based on Single Sliding Mode Observer," IEEE Transactions on Transportation Electrification, vol. 6, no. 2, pp. 679-689, Jun. 2020, doi: 10.1109/TTE.2020.2993950.

[11] Z. Ping, T. Wang, Y. Huang, H. Wang, J.-G. Lu, and Y. Li, "Internal Model Control of PMSM Position Servo System: Theory and Experimental Results," IEEE Transactions on Industrial Informatics, vol. 16, no. 4, pp. 2202-2211, Apr. 2020, doi: 10.1109/TII.2019.2935248.

[12] L. Qu, W. Qiao, and L. Qu, “An Enhanced Linear Active Disturbance Rejection Rotor Position Sensorless Control for Permanent Magnet Synchronous Motors," IEEE Transactions on Power Electronics, vol. 35, no. 6, pp. 6175-6184, Jun. 2020, doi: 10.1109/TPEL.2019.2953162.

[13] C. Lai et al., "PMSM Drive System Efficiency Optimization Using a Modified Gradient Descent Algorithm with Discretized Search Space," IEEE Transactions on Transportation Electrification, pp. 1-1, 2020, doi: 10.1109/TTE.2020.3004463.

[14] M. Rivera and J. Rodriguez, "Guest Editorial: Special Section on Predictive Control in Power Electronics, Electrical Drives and Industrial Applications," IEEE Journal of Emerging and Selected Topics in Power Electronics, vol. 6, no. 4, pp. 1638-1640, Dec. 2018, doi: 10.1109/JESTPE.2018.2877565.

[15] S. Kouro, P. Cortes, R. Vargas, U. Ammann, and J. Rodriguez, "Model Predictive Control-A Simple and Powerful Method to Control Power Converters," IEEE Transactions on Industrial Electronics, vol. 56, no. 6, pp. 1826-1838, Jun. 2009, doi: 10.1109/TIE.2008.2008349.

[16] J. Rodriguez et al., "State of the Art of Finite Control Set Model Predictive Control in Power Electronics," IEEE Transactions on Industrial Informatics, vol. 9, no. 2, pp. 1003-1016, May 2013, doi: 10.1109/TII.2012.2221469.

[17] K.-J. Lee, B.-G. Park, R.-Y. Kim, and D.-S. Hyun, "Robust Predictive Current Controller Based on a Disturbance Estimator in a Three-Phase Grid-Connected Inverter," IEEE Transactions on Power Electronics, vol. 27, no. 1, pp. 276-283, Jan. 2012, doi: 10.1109/TPEL.2011.2157706.

[18] Y. Zhang and W. Xie, "Low Complexity Model Predictive Control-Single Vector-Based Approach," IEEE Trans. Power Electron., vol. 29, no. 10, pp. 5532-5541, Oct. 2014, doi: 10.1109/TPEL.2013.2291005.
[19] "Predictive Control of Permanent Magnet Synchronous Motors," in Predictive Control of Power Converters and Electrical Drives, Chichester, UK: John Wiley \& Sons, Ltd, 2012, pp. 133-144.

[20] A. Kiselev, G. Catuogno, A. Kuznietsov, and R. Leidhold, "Finite Control Set MPC for Open-Phase Fault Tolerant Control of PM Synchronous Motor Drives," IEEE Transactions on Industrial Electronics, pp. 1-1, 2019, doi: 10.1109/TIE.2019.2931285.

[21] X. Zhang, L. Zhang, and Y. Zhang, "Model Predictive Current Control for PMSM Drives With Parameter Robustness Improvement," IEEE Transactions on Power Electronics, vol. 34, no. 2, pp. 1645-1657, Feb. 2019, doi: 10.1109/TPEL.2018.2835835.

[22] Y. Zhang, D. Xu, and L. Huang, "Generalized Multiple-Vector-Based Model Predictive Control for PMSM Drives," IEEE Trans. Ind. Electron., vol. 65, no. 12, pp. 9356-9366, Dec. 2018, doi: 10.1109/TIE.2018.2813994.

[23] Q. Wang, M. Rivera, J. A. Riveros, and P. Wheeler, "Modulated Model Predictive Current Control for PMSM Operating With Three-level NPC Inverter," in 2019 IEEE 15th Brazilian Power Electronics Conference and 5th IEEE Southern Power Electronics Conference (COBEP/SPEC), Dec. 2019, pp. 1-5, doi: 10.1109/COBEP/SPEC44138.2019.9065401.

[24] L. Tarisciotti, P. Zanchetta, A. Watson, J. C. Clare, M. Degano, and S. Bifaretti, "Modulated Model Predictive Control for a Three-Phase Active Rectifier," IEEE Transactions on Industry Applications, vol. 51, no. 2, pp. 1610-1620, Mar. 2015, doi: 10.1109/TIA.2014.2339397.

[25] M. Rivera et al., "A modulated model predictive control scheme for a two-level voltage source inverter," in 2015 IEEE International Conference on Industrial Technology (ICIT), Mar. 2015, pp. 2224-2229, doi: 10.1109/ICIT.2015.7125425.

[26] X. Li et al., "A Modulated Model Predictive Control Scheme for the Brushless Doubly Fed Induction Machine," IEEE Journal of Emerging and Selected Topics in Power Electronics, vol. 6, no. 4, pp. 1681-1691, Dec. 2018, doi: 10.1109/JESTPE.2018.2865668.

[27] L. Tarisciotti, P. Zanchetta, A. Watson, S. Bifaretti, and J. C. Clare, "Modulated model predictive control for a seven-level cascaded h-bridge back-to-back converter," IEEE Transactions on Industrial Electronics, vol. 61, no. 10, pp. 5375-5383, 2014, doi: 10.1109/TIE.2014.2300056.

[28] L. Tarisciotti et al., "Modulated Predictive Control for Indirect Matrix Converter," IEEE Transactions on Industry Applications, vol. 53, no. 5, pp. 4644-4654, Sep. 2017, doi: 10.1109/TIA.2017.2699666.

[29] M. Rivera et al., "Modulated Model Predictive Control (M2PC) with fixed switching frequency for an NPC converter," in 2015 IEEE 5th International Conference on Power Engineering, Energy and Electrical Drives (POWERENG), Riga, Latvia, May 2015, pp. 623-628, doi: 10.1109/PowerEng.2015.7266389.

[30] F. Donoso, A. Mora, R. Cárdenas, A. Angulo, D. Sáez, and M. Rivera, "Finite-Set Model-Predictive Control Strategies for a 3L-NPC Inverter Operating With Fixed Switching Frequency," IEEE Transactions on Industrial Electronics, vol. 65, no. 5, pp. 3954-3965, May 2018, doi: 10.1109/TIE.2017.2760840.

[31] C. Xia, T. Liu, T. Shi, and Z. Song, "A Simplified Finite-Control-Set Model-Predictive Control for Power Converters," IEEE Transactions on Industrial Informatics, vol. 10, no. 2, pp. 991-1002, May 2014, doi: 10.1109/TII.2013.2284558. 